\title{
ON TWO NEW MULTIDIMENSIONAL INTEGRAL INEQUALITIES OF THE HILBERT TYPE
}

\author{
B. G. PACHPATTE
}

\begin{abstract}
The main aim of this paper is to establish two new multidimensional integral inequalities similar to the integral analogue of the well known Hilbert's inequality by using elementary analysis.
\end{abstract}

\section{Introduction}

The integral analogue of the most celebrated Hilbert's double series theorem can be stated as follows (see [1, p. 226]).

Theorem H. If $p>1, p^{\prime}=p /(p-1)$ and

$$
\int_{0}^{\infty} f^{p}(x) d x \leq A, \int_{0}^{\infty} g^{p^{\prime}}(y) d y \leq B
$$

then

$$
\int_{0}^{\infty} \int_{0}^{\infty} \frac{f(x) g(y)}{x+y} d x d y<\frac{\pi}{\sin (\pi / p)} A^{1 / p} B^{1 / p^{\prime}}
$$

unless $f \equiv 0$ or $g \equiv 0$.

The Hilbert's double series theorem (see [1, p. 226]) and its integral analogue given in Theorem $\mathrm{H}$ led to a great many papers which deals with alternative proofs, various generalizations, numerous variants and applications in analysis. A survey of some of the earlier developments of this kind of inequalities and many important applications in analysis can be found in [1, Chapter IX]. Recently, in [4-10] the present author has established some new inequalities similar to Hilbert's double series inequality and its integral analogue which are of independent interest. The main purpose of this paper is to establish two new integral inequalities similar to the integral analogue of the Hilbert's inequality involving functions of two and many independent variables. The analysis used

Received April 21, 1999.

2000 Mathematics Subject Classification. 26D10, 26D15.

Key words and phrases. Multidimensional integral inequalities, Hilbert's inequality, integral analogue of the Hilbert's inequality, functions of two and many independent variables, Schwarz inequality. 
in the proofs is elementary and our results provides new estimates on inequalities of this type.

\section{Statement of Results}

In what follows we denote by $R$ set of real numbers. Let $I_{x}=[0, x), I_{y}=[0, y)$, $I_{z}=[0, z), I_{w}=[0, w), I=[0, \infty), I_{0}=(0, \infty)$ denotes the subintervals of $R$, where $x, y, z, w$ are the elements of $I_{0}$ and let $\Delta_{1}=I_{x} \times I_{y}$ and $\Delta_{2}=I_{z} \times I_{w}$. For the function $u(s, t)$ the partial derivatives $\frac{\partial}{\partial s} u(s, t)$ and $\frac{\partial}{\partial t} u(s, t)$ are denoted by $D_{1} u(s, t)$ and $D_{2} u(s, t)$ respectively. The higher order derivatives of $u(s, t)$ can be denoted similarly. We denote by $H(\Delta)$, where $\Delta=I \times I$, the class of functions $u(s, t) \in C^{(n-1, m-1)}(\Delta)$ such that $D_{1}^{i} u(0, t)=0,0 \leq i \leq n-1, t \in I, D_{2}^{j} u(s, 0)=0,0 \leq j \leq m-1, s \in I$, and $D_{1}^{n} D_{2}^{m-1} u(s, t)$ and $D_{1}^{n-1} D_{2}^{m} u(s, t)$ are absolutely continuous on $I \times I$.

Let $E$ and $F$ be bounded domains in $I^{n}$ defined by $E=\prod_{i=1}^{n}\left[0, a_{i}\right)$ and $F=$ $\prod_{i=1}^{n}\left[0, b_{i}\right)$, where $a_{i}, b_{i}$ are the elements of $I_{0}$. Let $x=\left(x_{1}, \ldots, x_{n}\right), y=\left(y_{1}, \ldots, y_{n}\right)$ denote the variable points in $E$ and $F$ respectively and $d x=d x_{1} \cdots d x_{n}$ and $d y=$ $d y_{1} \cdots d y_{n}$. For any continuous real-valued functions $u$ and $v$ defined on $E$ and $F$ respectively, we denote by $\int_{E} u(\xi) d \xi$ and $\int_{F} v(\eta) d \eta$ the $n$-fold integrals $\int_{0}^{a_{1}} \cdots \int_{0}^{a_{n}} u\left(\xi_{1}, \ldots, \xi_{n}\right)$ $\times d \xi_{1} \cdots d \xi_{n}$ and $\int_{0}^{b_{1}} \cdots \int_{0}^{b_{n}} v\left(\eta_{1}, \ldots, \eta_{n}\right) d \eta_{1} \cdots d \eta_{n}$ respectively. For any $x \in E, y \in F$ we denote by $\int_{E_{x}} u(s) d s$ and $\int_{F_{y}} v(t) d t$ the $n$-fold integrals $\int_{0}^{x_{1}} \cdots \int_{0}^{x_{n}} u\left(s_{1}, \ldots, s_{n}\right) d s_{1}$ $\cdots d s_{n}$ and $\int_{0}^{y_{1}} \cdots \int_{0}^{y_{n}} v\left(t_{1}, \ldots, t_{n}\right) d t_{1} \cdots d t_{n}$ respectively. We denote by $G(E)$ and $G(F)$ respectively the classes of continuous functions $u: E \rightarrow R$ and $v: F \rightarrow R$ for which the partial derivatives $D_{1} \cdots D_{n} u(x)$ and $D_{1} \cdots D_{n} v(y)$ exist and such that

$$
\begin{gathered}
u\left(0, x_{2}, \ldots, x_{n}\right)=u\left(x_{1}, 0, x_{3}, \ldots, x_{n}\right)=\cdots=u\left(x_{1}, \ldots, x_{n-1}, 0\right)=0, \\
v\left(0, y_{2}, \ldots, y_{n}\right)=v\left(y_{1}, 0, y_{3}, \ldots, y_{n}\right)=\cdots=v\left(y_{1}, \ldots, y_{n-1}, 0\right)=0
\end{gathered}
$$

where $D_{i}=\frac{\partial}{\partial x_{i}}$ for $i=i, \ldots, n$.

Our first theorem deals with an inequality similar to the integral analogue of the Hilbert's inequality involving functions of two independent variables and their higher order partial derivatives.

Theorem 1. Let $u(s, t) \in H\left(\Delta_{1}\right)$ and $v(k, r) \in H\left(\Delta_{2}\right)$. Then for $0 \leq i \leq n-1$, $0 \leq j \leq m-1$, the following inequality holds

$$
\begin{aligned}
& \int_{0}^{x} \int_{0}^{y}\left(\int_{0}^{z} \int_{0}^{w} \frac{\left|D_{1}^{i} D_{2}^{j} u(s, t)\right|\left|D_{1}^{i} D_{2}^{j} v(k, r)\right|}{s^{2 n-2 i-1} t^{2 m-2 j-1}+k^{2 n-2 i-1} r^{2 m-2 j-1}} d k d r\right) d s d t \\
\leq & \frac{1}{2}\left[A_{i, j} B_{i, j}\right]^{2} \sqrt{x y z w}\left(\int_{0}^{x} \int_{0}^{y}(x-s)(y-t)\left|D_{1}^{n} D_{2}^{m} u(s, t)\right|^{2} d s d t\right)^{1 / 2} \\
& \times\left(\int_{0}^{z} \int_{0}^{w}(z-k)(w-r)\left|D_{1}^{n} D_{2}^{m} v(k, r)\right|^{2} d k d r\right)^{1 / 2} .
\end{aligned}
$$


for $x, y, z, w$ in $I_{0}$, where

$$
\begin{aligned}
A_{i, j} & =\frac{1}{(n-i-1) !(m-j-1) !} \\
B_{i, j} & =\frac{1}{(2 n-2 i-1)(2 m-2 j-1)}
\end{aligned}
$$

Remark 1. If we take $i=0, j=0$ in (1), then we get the following inequality

$$
\begin{aligned}
& \int_{0}^{x} \int_{0}^{y}\left(\int_{0}^{z} \int_{0}^{w} \frac{|u(s, t)||v(k, r)|}{s^{2 n-1} t^{2 m-1}+k^{2 n-1} r^{2 m-1}} d k d r\right) d s d t \\
\leq & \frac{1}{2}\left[A_{0,0} B_{0,0}\right]^{2} \sqrt{x y z w}\left(\int_{0}^{x} \int_{0}^{y}(x-s)(y-t)\left|D_{1}^{n} D_{2}^{m} u(s, t)\right|^{2} d s d t\right)^{1 / 2} \\
& \times\left(\int_{0}^{z} \int_{0}^{w}(z-k)(w-r)\left|D_{1}^{n} D_{2}^{m} v(k, r)\right|^{2} d k d r\right)^{1 / 2} .
\end{aligned}
$$

Furthermore, if we take $n=1, m=1$ in (4), then we get the following inequality recently established by Pachpatte in [9]

$$
\begin{aligned}
& \int_{0}^{x} \int_{0}^{y}\left(\int_{0}^{z} \int_{0}^{w} \frac{|u(s, t)||v(k, r)|}{s t+k r} d k d r\right) d s d t \\
\leq & \frac{1}{2} \sqrt{x y z w}\left(\int_{0}^{x} \int_{0}^{y}(x-s)(y-t)\left|D_{1} D_{2} u(s, t)\right|^{2} d s d t\right)^{1 / 2} \\
& \times\left(\int_{0}^{z} \int_{0}^{w}(z-k)(w-r)\left|D_{1} D_{2} v(k, r)\right|^{2} d k d r\right)^{1 / 2}
\end{aligned}
$$

Another interesting inequality similar to the integral analogue of the Hilbert's inequality involving functions of several variables and their partial derivatives is given in the following theorem.

Theorem 2. Let $u(x) \in G(E)$ and $v(y) \in G(F)$. Then the following inequality holds

$$
\begin{aligned}
& \int_{E}\left(\int_{F} \frac{|u(x)||v(y)|}{\prod_{i=1}^{n} x_{i}+\prod_{i=1}^{n} y_{i}} d y\right) d x \\
\leq & \frac{1}{2}\left(\prod_{i=1}^{n} a_{i}\right)^{1 / 2}\left(\prod_{i=1}^{n} b_{i}\right)^{1 / 2} \times\left(\int_{E} \prod_{i=1}^{n}\left(a_{i}-x_{i}\right)\left|D_{1} \cdots D_{n} u(x)\right|^{2} d x\right)^{1 / 2} \\
& \times\left(\int_{F} \prod_{i=1}^{n}\left(b_{i}-y_{i}\right)\left|D_{1} \cdots D_{n} v(y)\right|^{2} d y\right)^{1 / 2}
\end{aligned}
$$

Remark 2. In the special case when $n=2$, the inequality (6) reduces to the inequality (5) with suitable changes, which is recently established by the present author in $[9]$. 


\section{Proof of Theorem 1}

From the hypotheses we have the following identities (see [11]])

$$
\begin{aligned}
& D_{1}^{i} D_{2}^{j} u(s, t)=A_{i, j} \int_{0}^{s} \int_{0}^{t}(s-\xi)^{n-i-1}(t-\eta)^{m-j-1} D_{1}^{n} D_{2}^{m} u(\xi, \eta) d \xi d \eta, \\
& D_{1}^{i} D_{2}^{j} v(k, r)=A_{i, j} \int_{0}^{k} \int_{0}^{r}(k-\sigma)^{n-i-1}(r-\tau)^{m-j-1} D_{1}^{n} D_{2}^{m} v(\sigma, \tau) d \sigma d \tau,
\end{aligned}
$$

for $(s, t) \in \Delta_{1},(k, r) \in \Delta_{2}$. From (7) and (8) and using Schwarz inequality we observe that

$$
\left|D_{1}^{i} D_{2}^{j} u(s, t)\right| \leq A_{i, j} B_{i, j}\left[s^{2 n-2 i-1} t^{2 m-2 j-1}\right]^{1 / 2} \times\left(\int_{0}^{s} \int_{0}^{t}\left|D_{1}^{n} D_{2}^{m} u(\xi, \eta)\right|^{2} d \xi d \eta\right)^{1 / 2},
$$

and

$$
\left|D_{1}^{i} D_{2}^{j} v(k, r)\right| \leq A_{i, j} B_{i, j}\left[k^{2 n-2 i-1} r^{2 m-2 j-1}\right]^{1 / 2} \times\left(\int_{0}^{k} \int_{0}^{r}\left|D_{1}^{n} D_{2}^{m} v(\sigma, \tau)\right|^{2} d \sigma d \tau\right)^{1 / 2} .
$$

From (9) and (10) and using the elementary inequality $c^{1 / 2} d^{1 / 2} \leq \frac{1}{2}(c+d)$, (for $c, d$ nonnegative reals) we observe that

$$
\begin{aligned}
\frac{\left|D_{1}^{i} D_{2}^{j} u(s, t)\right|\left|D_{1}^{i} D_{2}^{j} v(k, r)\right|}{s^{2 n-2 i-1} t^{2 m-2 j-1}+k^{2 n-2 i-1} r^{2 m-2 j-1}} \leq & \frac{1}{2}\left[A_{i, j} B_{i, j}\right]^{2}\left(\int_{0}^{s} \int_{0}^{t} \mid D_{1}^{n} D_{2}^{m} u(\xi, \eta) d \xi d \eta\right)^{1 / 2} \\
& \times\left(\int_{0}^{k} \int_{0}^{r}\left|D_{1}^{n} D_{2}^{m} v(\sigma, \tau)\right|^{2} d \sigma d \tau\right)^{1 / 2} .
\end{aligned}
$$

Integrating both sides of (11) first over $r$ from 0 to $w$ and over $k$ from 0 to $z$ and then integrating both sides of the resulting inequality over $t$ from 0 to $y$ and over $s$ from 0 to $x$ and using Schwarz inequality and Fubini's theorem we observe that

$$
\begin{aligned}
& \int_{0}^{x} \int_{0}^{y}\left(\int_{0}^{z} \int_{0}^{w} \frac{\left|D_{1}^{i} D_{2}^{j} u(s, t)\right|\left|D_{1}^{i} D_{2}^{j} v(k, r)\right|}{s^{2 n-2 i-1} t^{2 m-2 j-1}+k^{2 n-2 i-1} r^{2 m-2 j-1}} d k d r\right) d s d t \\
\leq & \frac{1}{2}\left[A_{i, j} B_{i, j}\right]^{2}\left(\int_{0}^{x} \int_{0}^{y}\left(\int_{0}^{s} \int_{0}^{t}\left|D_{1}^{n} D_{2}^{m} u(\xi, \eta)\right|^{2} d \xi d \eta\right)^{1 / 2} d s d t\right) \\
& \times\left(\int_{0}^{z} \int_{0}^{w}\left(\int_{0}^{k} \int_{0}^{r}\left|D_{1}^{n} D_{2}^{m} v(\sigma, \tau)\right|^{2} d \sigma d \tau\right)^{1 / 2} d k d r\right) \\
\leq & \frac{1}{2}\left[A_{i, j} B_{i, j}\right]^{2} \sqrt{x y}\left(\int_{0}^{x} \int_{0}^{y}\left(\int_{0}^{s} \int_{0}^{t}\left|D_{1}^{n} D_{2}^{m} u(\xi, \eta)\right|^{2} d \xi d \eta\right) d s d t\right)^{1 / 2} \\
& \times \sqrt{z w}\left(\int_{0}^{z} \int_{0}^{w}\left(\int_{0}^{k} \int_{0}^{r}\left|D_{1}^{n} D_{2}^{m} v(\sigma, \tau)\right|^{2} d \sigma d \tau\right) d k d r\right)^{1 / 2} \\
= & \frac{1}{2}\left[A_{i, j} B_{i, j}\right]^{2} \sqrt{x y z w}\left(\int_{0}^{x} \int_{0}^{y}(x-s)(y-t)\left|D_{1}^{n} D_{2}^{m} u(s, t)\right|^{2} d s d t\right)^{1 / 2} \\
& \times\left(\int_{0}^{z} \int_{0}^{w}(z-k)(w-r)\left|D_{1}^{n} D_{2}^{m} v(k, r)\right|^{2} d k d r\right)^{1 / 2} .
\end{aligned}
$$


This is the desired inequality in (1) and the proof is complete.

\section{Proof of Theorem 2}

From the hypotheses we have the following identities (see [12])

$$
\begin{aligned}
& u(x)=\int_{E_{x}} D_{1} \cdots D_{n} u(s) d s, \\
& v(y)=\int_{F_{y}} D_{1} \cdots D_{n} v(t) d t,
\end{aligned}
$$

for $x \in E$ and $y \in F$. From (12) and (13) and using Schwarz inequality we have

$$
\begin{aligned}
|u(x)| & \leq\left(\prod_{i=1}^{n} x_{i}\right)^{1 / 2}\left(\int_{E_{x}}\left|D_{1} \cdots D_{n} u(s)\right|^{2} d s\right)^{1 / 2}, \\
|v(y)| & \leq\left(\prod_{i=1}^{n} y_{i}\right)^{1 / 2}\left(\int_{F_{y}}\left|D_{1} \cdots D_{n} v(t)\right|^{2} d t\right)^{1 / 2} .
\end{aligned}
$$

From (14), (15) and using the elementary inequality $c^{1 / 2} d^{1 / 2} \leq \frac{1}{2}(c+d)$, (for $c, d$ nonnegative reals) and rewriting we observe that

$$
\frac{|u(x)||v(y)|}{\prod_{i=1}^{n} x_{i}+\prod_{i=1}^{n} y_{i}} \leq \frac{1}{2}\left(\int_{E_{x}}\left|D_{1} \cdots D_{n} u(s)\right|^{2} d s\right)^{1 / 2} \times\left(\int_{F_{y}}\left|D_{1} \cdots D_{n} v(t)\right|^{2} d t\right)^{1 / 2} .
$$

Integrating both sides of (16) first over $F$ and then integrating both sides of the resulting inequality over $E$ and using Schwarz inequality and Fubini's theorem we observe that

$$
\begin{aligned}
& \int_{E}\left(\int_{F} \frac{|u(x)||v(y)|}{\prod_{i=1}^{n} x_{i}+\prod_{i=1}^{n} y_{i}} d y\right) d x \leq \frac{1}{2}\left(\int_{E}\left(\int_{E_{x}}\left|D_{1} \cdots D_{n} u(s)\right|^{2} d s\right)^{1 / 2} d x\right) \\
& \times\left(\int_{F}\left(\int_{F_{y}}\left|D_{1} \cdots D_{n} v(t)\right|^{2} d t\right)^{1 / 2} d y\right) \\
\leq & \frac{1}{2}\left(\prod_{i=1}^{n} a_{i}\right)^{1 / 2}\left(\int_{E}\left(\int_{E_{x}}\left|D_{1} \cdots D_{n} u(s)\right|^{2} d s\right) d x\right)^{1 / 2} \\
& \times\left(\prod_{i=1}^{n} b_{i}\right)^{1 / 2}\left(\int_{F}\left(\int_{F_{y}}\left|D_{1} \cdots D_{n} v(t)\right|^{2} d t\right) d y\right)^{1 / 2} \\
= & \frac{1}{2}\left(\prod_{i=1}^{n} a_{i}\right)^{1 / 2}\left(\prod_{i=1}^{n} b_{i}\right)^{1 / 2} \times\left(\int_{E} \prod_{i=1}^{n}\left(a_{i}-x_{i}\right)\left|D_{1} \cdots D_{n} u(x)\right|^{2} d x\right)^{1 / 2} \\
& \times\left(\int_{F} \prod_{i=1}^{n}\left(b_{i}-y_{i}\right)\left|D_{1} \cdots D_{n} v(y)\right|^{2} d y\right)^{1 / 2} .
\end{aligned}
$$


This is the required inequality in (6) and the proof is complete.

Remark 3. If we apply the elementary inequality $c^{1 / 2} d^{1 / 2} \leq \frac{1}{2}(c+d)$, (for $c, d$ nonnegative reals) on the right hand sides of (1) and (6), then we get respectively the following new inequalities

$$
\begin{aligned}
& \int_{0}^{x} \int_{0}^{y}\left(\int_{0}^{z} \int_{0}^{w} \frac{\left|D_{1}^{i} D_{2}^{j} u(s, t)\right|\left|D_{1}^{i} D_{2}^{j} v(k, r)\right|}{s^{2 n-2 i-1} t^{2 m-2 j-1}+k^{2 n-2 i-1} r^{2 m-2 j-1}} d k d r\right) d s d t \\
\leq & \frac{1}{4}\left[A_{i, j} B_{i, j}\right]^{2} \sqrt{x y z w} \times\left[\int_{0}^{x} \int_{0}^{y}(x-s)(y-t)\left|D_{1}^{n} D_{2}^{m} u(s, t)\right|^{2} d s d t\right. \\
& \left.+\int_{0}^{z} \int_{0}^{w}(z-k)(w-r)\left|D_{1}^{n} D_{2}^{m} v(k, r)\right|^{2} d k d r\right],
\end{aligned}
$$

and

$$
\begin{aligned}
\int_{E}\left(\int_{F} \frac{|u(x)||v(y)|}{\prod_{i=1}^{n} x_{i}+\prod_{i=1}^{n} y_{i}} \leq\right. & \frac{1}{4}\left(\prod_{i=1}^{n} a_{i}\right)^{1 / 2}\left(\prod_{i=1}^{n} b_{i}\right)^{1 / 2} \times\left[\int_{E} \prod_{i=1}^{n}\left(a_{i}-x_{i}\right)\left|D_{1} \cdots D_{n} u(x)\right|^{2} d x\right. \\
& \left.+\int_{F} \prod_{i=1}^{n}\left(b_{i}-y_{i}\right)\left|D_{1} \cdots D_{n} v(y)\right|^{2} d y\right] .
\end{aligned}
$$

We note that the inequalities established in $(1),(6),(17),(18)$ can be considered as further extensions of the inequalities recently established by the present author in $[9,10]$. For a number of new inequalities similar to Hilbert's inequality, we refer the interested readers to the recent papers [4-10]. In fact our results are obtained by using quite elementary analysis and the bounds obtained in the inequalities are new and can not be compared with the bound given in the integral analogue of the Hilbert's inequality.

\section{References}

[1] G. H. Hardy, J. E. Littlewood and G. Polya, Inequalities, Cambridge University Press, Cambridge, 1934.

[2] G. Mingze, On Hilbert's inequality and its applications, J. Math. Anal. Appl., 212(1997), 316-323.

[3] D. S. Mitrinović and J. E. Pečarić, On inequalities of Hilbert and Widder, Proc. Edinburgh Math. Soc., 34(1991), 411-414.

[4] B. G. pachpatte, On some new inequalities similar to Hilbert's inequality, J. Math. Anal. Appl., 226(1998), 166-179.

[5] B. G. Pachpatte, A note on Hilbert type inequality, Tamkang J. Math., 29(1998), 293-298.

[6] B. G. Pachpatte, A note on an inequality of Hilbert type, Octogon Math. Mag., to appear.

[7] B. G. Pachpatte, Inequalities similar to the integral analogue of Hilbert's inequality, Tamkang J. Math., 30(1999), 139-146.

[8] B. G. Pachpatte, On an inequality similar to Hilbert's inequality, Bull. Inst. Polyt. Iasi, to appear. 
[9] B. G. Pachpatte, On a new inequality analogous to Hilbert's inequality, Radovi Math., to appear.

[10] B. G. Pachpatte, Inequalities similar to certain extensions of Hilbert's inequality, J. Math. Anal. Appl., to appear.

[11] B. G. Pachpatte, Existence and uniqueness of solutions of higher order Hyperbolic partial differential equations, Chinese J. Math., 17(1989), 181-189.

[12] B. G. Pachpatte, Opial type inequality in several variables, Tamkang J. Math., 22(1991), $7-11$.

Department of mathematics, Marathwada University, Aurangabad 431 004, (Maharashtra) India. 